\title{
Adolescent blood pressure hyperreactors have a higher reactive hyperemic index at the fingertip
}

\author{
Thomas Radtke $\cdot$ Prisca Eser • Susi Kriemler • \\ Hugo Saner $\cdot$ Matthias Wilhelm
}

Received: 29 May 2013 / Accepted: 20 September 2013 / Published online: 29 September 2013

(C) Springer-Verlag Berlin Heidelberg 2013

\begin{abstract}
Purpose The use of peripheral arterial tonometry (PAT) to assess microvascular endothelial function is getting increasingly popular in the pediatric population. The aim of the present study was to examine the impact of blood pressure (BP) hyperreactivity on microvascular hyperemic reaction in healthy, normotensive adolescents.

Methods Fifty-two adolescents with a mean age of $14.5 \pm 0.7$ years were investigated. Microvascular endothelial function was assessed at rest and after exhaustive cycling exercise using PAT to determine the reactive hyperemic index (RHI, primary endpoint). Hyperreactors were identified according to their cardiovascular response to a cold pressor test. Indices of autonomic tone were assessed with an ambulatory electrocardiograph and cardiopulmonary exercise testing was performed on a cycle ergometer. Physical activity was measured using accelerometry. ANOVA with repeated measures and Tukey HSD post hoc tests were applied to determine differences between BP hyperreactors and controls (normal reactors).

Results Sixteen adolescents were identified as hyperreactors and matched with regard to age, sex, pubertal status, and height with 16 normal reactors. At rest,
\end{abstract}

Communicated by Massimo Pagani.

T. Radtke $(\varangle) \cdot$ P. Eser $\cdot$ H. Saner $\cdot$ M. Wilhelm Division of Cardiovascular Prevention, Rehabilitation and Sports Medicine, University Clinic for Cardiology, Inselspital, University Hospital and University of Bern, 3010 Bern, Switzerland

e-mail: thomas.radtke@insel.ch

S. Kriemler

Institute of Social and Preventive Medicine, University of Zurich,

Zurich, Switzerland hyperreactors had a significantly higher RHI than normal reactors $(2.1 \pm 0.4$ vs. $1.6 \pm 0.4, P=0.003)$. After exhaustive cycling exercise, the differences in RHI were abolished. No differences between these two groups existed in physical activity levels, exercise capacity and indices of autonomic tone.

Conclusions Our data suggest that normotensive hyperreactors have a markedly higher RHI compared to normal reactors. Future studies using PAT to assess microvascular endothelial function should bare in mind the large effect of vascular hyperreactivity.

Keywords Cardiovascular hyperreactivity - Peripheral arterial tonometry $\cdot$ Hypertension · Endothelial function · Physical activity

\section{Introduction}

There is growing interest in assessing microvascular endothelial function as part of the cardiovascular risk assessment in adults (Hamburg et al. 2011), and also in the pediatric population (Bruyndonckx et al. 2013; Haller et al. 2007; Mahmud et al. 2009; Radtke et al. 2012, 2013b). Peripheral arterial tonometry (PAT) is a non-invasive and operatorindependent technique, used to assess the nitric oxide (NO) bioavailability as a measure of human endothelial function (Nohria et al. 2006). Recently, Hamburg et al. (2011) have investigated the prevalence and risk factors associated with impaired microvascular endothelial function using PAT in a large healthy cohort. Unexpectedly, a high BP was associated with a lower prevalence of abnormal microvascular endothelial function. It was speculated that a high blood pressure (BP) may affect tone and structure of the microvascular bed, thus leading to higher hyperemic pulse wave 
amplitudes (PWA) in the digit and consequently enhanced microvascular hyperemic reaction (Hamburg et al. 2011). This indicates that the morphological and functional factors influencing the microvascular hyperemic response are not at all clear yet.

Increased cardiovascular reactivity to stress, such as the cold pressor test (CPT), has been suggested to be a predictor of the development of hypertension (Hines and Brown 1936). The cardiovascular response to the CPT in school children has first been described by Hines (1937). Later, several longitudinal studies have observed that BP hyperreactivity to the CPT increases the risk for future hypertension (Barnett et al. 1963; Flaa et al. 2008; Kasagi et al. 1995; Menkes et al. 1989; Wood et al. 1984), suggesting that an altered physiology is already present at a young age (Menkes et al. 1989). The autonomic nervous system plays a central role in the pathogenesis of hypertension (Abboud et al. 2012; Gamboa et al. 2012). Multiple pathways are involved, and NO, synthesized by the vascular endothelium, is thought to play a pivotal role in the regulation of vascular tone and the development of hypertension (Forte et al. 1997).

The aim of this study was: (1) To identify hyperreactors among a random group of healthy adolescents and (2) to determine the impact of BP hyperreactivity as assessed by a CPT on microvascular endothelial function at rest and (3) to assess the contribution of sympathetic activity on microvascular reactivity by means of a maximal exercise bout. We hypothesized that BP hyperreactors have increased microvascular reactivity at rest, expressed as a higher reactive hyperemic index (RHI, primary endpoint) and PAT ratio compared to normal reactors. Moreover, it was hypothesized that the increased microvascular reactivity in BP hyperreactors at rest would be mitigated after a single bout of exhaustive exercise.

\section{Materials and methods}

\section{Participant characteristics}

Healthy adolescents (aged 14-16 years) were recruited from an elementary school in Olten, Switzerland. We excluded subjects with juvenile rheumatoid arthritis or vascular diseases (Reynaud's or Kawasaki's disease), hypertension (National High Blood Pressure Education Program Working Group on High Blood Pressure in Children and Adolescents 2004), obesity (Fernandez et al. 2004), diabetes, depression, smoking and intake of any vasoactive medication. Written informed consent was obtained from all participating subjects and parents/caregivers. Ethical approval was obtained from the cantonal ethical committee of Bern, Switzerland.
Experimental design

Subjects visited a temporary laboratory at school, once to complete a number of physiological examinations. Microvascular endothelial function and the CPT were performed twice: at rest after an overnight fast between 8 a.m. and 10 a.m. (session I) and again after an exhaustive cardiopulmonary exercise test (session II). The two test sessions were separated by a $1 \mathrm{~h}$ break during which participants received a standardized snack containing a chocolate milk drink and a granola bar.

Anthropometric and hemodynamic measurements

Body mass was measured with light clothing to the nearest $0.1 \mathrm{~kg}$ using a digital-balanced scale and stature to the nearest $0.5 \mathrm{~cm}$ using a wall-mounted stadiometer. Body mass index (BMI) was calculated as body mass divided by stature squared $\left(\mathrm{kg} \mathrm{m}^{-2}\right)$. Waist circumference was measured twice in standing position at the midpoint between the last rib and the iliac crest using an anthropometric tape. Skinfold thickness was measured in triplicate on the right side of the body and to the nearest $0.2 \mathrm{~mm}$ with a Harpenden caliper. The sum of four sites (triceps, biceps, subscapular and suprailiacal) was calculated and the average of three measurements was used for analysis. Pubertal stage (Tanner 1-5) was assessed by use of a validated self-assessment tool (Morris and Udry 1980). Resting BP was measured in the sitting position 3 times at 2 min intervals at the right arm with an adequately sized cuff [Philips Small Adult (20.5-28.5 cm)] using an oscillometric device (Dinamap XL; Criticon Inc., Tampa, FL, USA). The mean of the three measurements was used for data analysis and z-transformed (National High Blood Pressure Education Program Working Group on High Blood Pressure in Children and Adolescents 2004). A 12-lead resting electrocardiograph (ECG) was performed to exclude those with increased risk for exercise-induced arrhythmias such as long Q-T syndrome.

Adapted cold pressor test

The CPT was performed to identify hyperreactors according to the method described by Hines and Brown (1936), e.g. subjects who responded with an increase of $\geq 15 \mathrm{mmHg}$ in systolic and/or diastolic BP at the end of cold-water immersion (CWI) (Kasagi et al. 1995; Menkes et al. 1989). The CPT tests were performed at rest (session I) and again $30 \mathrm{~min}$ after completion of an exhaustive cardiopulmonary exercise test on a cycle ergometer (session II). Figure 1 shows the detailed experimental protocol. In preparation for the CPT, subjects rested for $3 \mathrm{~min}$ in sitting position and then immersed their right hand in $37 \pm 1^{\circ} \mathrm{C}$ 


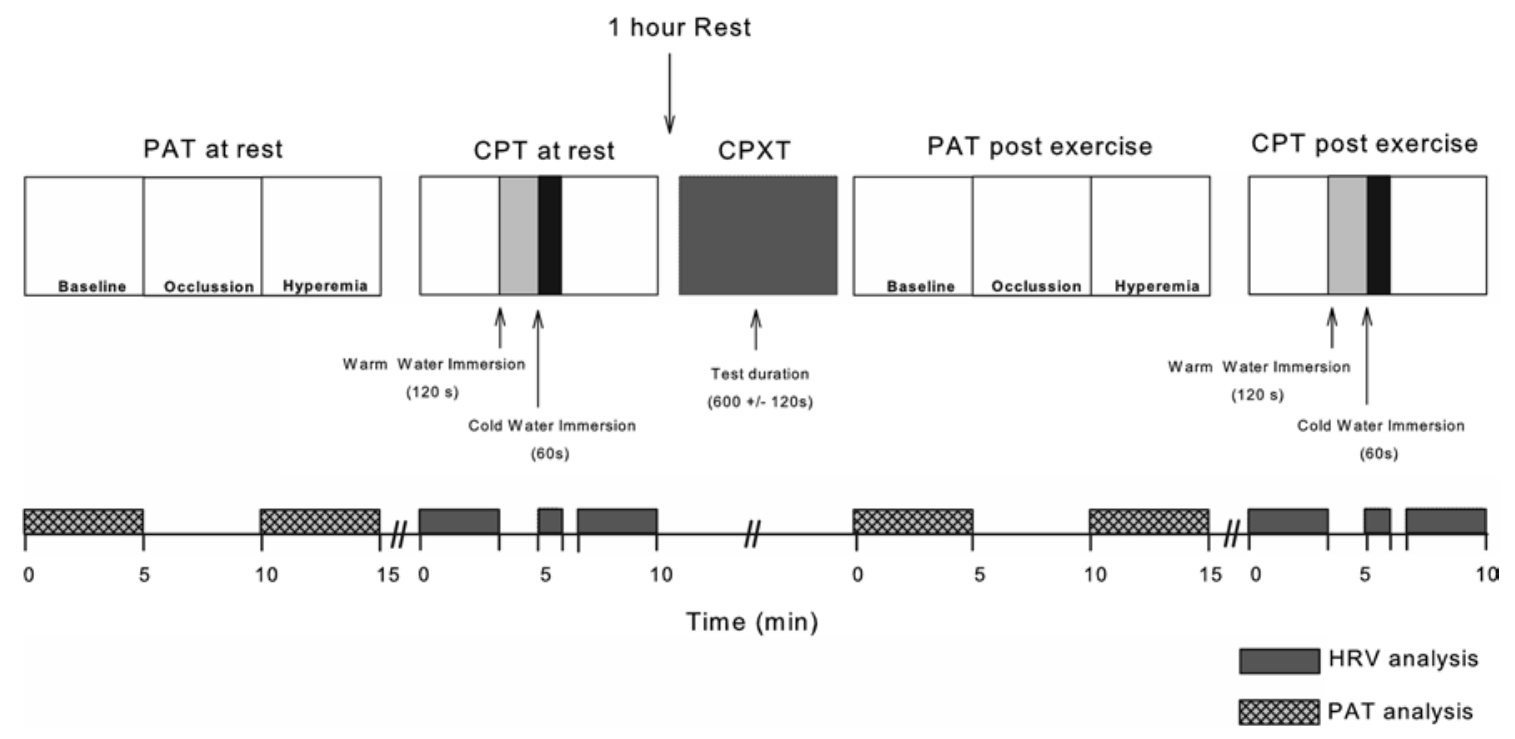

Fig. 1 Experimental study design. Peripheral arterial tonometry (PAT) and the cold pressor test (CPT) were performed twice, at rest (session I) and 30 min after a maximal cardiopulmonary exercise test

temperature controlled water for $2 \mathrm{~min}$. This procedure was chosen to guarantee identical pre-test conditions for all subjects. Subsequently, subjects placed their right hand up to and including the wrist into a custom made and temperature controlled box, containing $5 \pm 1{ }^{\circ} \mathrm{C}$ cold water for a period of 1 min. A water pump (JBL ProFlow Maxi 750) produced a continuous flow around the immersed hand. After CWI subjects rested for $4 \mathrm{~min}$. BP was measured in the arm, which hand was not immersed, one minute after warm water immersion, immediately after CWI and again 2 and 4 min after CWI.

Assessment of microvascular endothelial function

Microvascular endothelial function was measured at rest and immediately after exhaustive cycling exercise using PAT (Endo-PAT2000 ${ }^{\circledR}$, Itamar Medical Ltd., Israel) in a quiet, temperature controlled and light dimmed room (22$24{ }^{\circ} \mathrm{C}$ ). Participants were in a supine position with pneumatic fingertip probes placed on both index fingers and pulse wave amplitudes (PWA) were continuously recorded. Pulsatile arterial volume changes at rest and after induction of reactive hyperemia are recorded digitally as PWA over time. The PWA is defined as the difference between the highest and lowest point of the pulse wave (arbitrary unit). After a 5 min baseline measurement, an ischemic stimulus was induced over $5 \mathrm{~min}$ by cuff inflation $(\geq 220 \mathrm{mmHg}$ ) of the non-dominant upper arm which was maintained for 5 min. The BP cuff was then rapidly deflated and bilateral PWA's were recorded for $5 \mathrm{~min}$. The reactive hyperemic index (RHI) was generated by the PAT software program
(CPXT, session II, post-exercise). The boxes indicate the time segments during which the heart rate variability (HRV) and PAT data were analyzed

as the ratio of the average PWA over $60 \mathrm{~s}$ starting $90 \mathrm{~s}$ after cuff deflation to the average PWA measured at baseline, and then divided by the same ratio in the contra-lateral finger. Reproducibility and feasibility of this device has recently been demonstrated in healthy adolescents (Selamet Tierney et al. 2009). Ratios of post-occlusion amplitudes divided by baseline amplitudes were calculated for both sides and then the response of the occluded side was divided by the response of the non-occluded side (control finger), resulting in the PAT ratio. Recent work using PAT revealed that the time to peak response after cuff deflation occurred later in children and adolescents than in adults, suggesting that the peak response may not be optimally detected by the automated algorithm provided by the manufacturer that uses a fixed time interval (Chen et al. 2012). Therefore, we also visually analyzed the overall 5 min post-occlusion response curve using averages of each $30 \mathrm{~s}$ amplitude interval to determine the peak response as well as the time to peak. We defined the time to peak using the 'midpoint' of the corresponding $30 \mathrm{~s}$ average amplitude interval (e.g. including $15 \mathrm{~s}$ of this interval).

\section{Assessment of physical activity}

Physical activity (PA) measurements were obtained using a three axial accelerometer (GT3X, ActiGraph ${ }^{\circledR}$, Shalimar, FL, USA). The monitor was attached at the right hip with a belt and worn for eight consecutive days, except for water activities, contact sports and sleeping. The accelerometer was programmed to record PA data every $2 \mathrm{~s}$. Data from the accelerometers were downloaded and checked for spurious 
counts. Excessively high counts $(\geq 20,000)$ were removed from the analysis (Masse et al. 2005). Sustained periods of $\geq 20 \mathrm{~min}$ of continuous zero values were interpreted as missing. A minimum of 5 days including one weekend day with $\geq 9 \mathrm{~h}$ daily wear time were required for inclusion in the data analysis. Data were expressed as daily time spent at moderate-to-vigorous physical activity according to published cut-offs (Treuth et al. 2004).

\section{Assessment of cardiorespiratory fitness}

Cardiopulmonary exercise testing (CPXT) was performed on an electromagnetically braked cycle ergometer (Schiller 911 S, Schiller-Reomed AG, Dietikon, Switzerland) with online gas analysis until exhaustion. A progressive incremental cycle protocol was chosen to assure subjects achieved their limit of tolerance within $10 \pm 2$ min. Respiratory parameters were measured continuously in an open circuit system (CS 200, Schiller-Reomed AG, Dietikon, Switzerland). Heart rate and cardiac rhythm were continuously analyzed using a 12-lead ECG. All subjects received verbal encouragement to reach their maximal performance. Clinical signs of exhaustion and further objective criteria were used to confirm peak oxygen uptake $\left(\dot{V} \mathrm{O}_{2}\right.$ peak $)$, i.e.: a peak heart rate approaching 200 beats $\mathrm{min}^{-1}$, a respiratory exchange ratio $>1.10$, or oxygen plateau $<2 \mathrm{~mL} \mathrm{~kg}^{-1} \mathrm{~min}^{-1}$ increase in $\dot{V} \mathrm{O}_{2}$ with increasing work rate (Armstrong et al. 1991; Paridon et al. 2006). The highest 15 s averaged $\dot{V} \mathrm{O}_{2}$ achieved during the test was considered as $\dot{V} \mathrm{O}_{2}$ peak.

Assessment of autonomic nervous system activity

Heart rate and HRV as markers of autonomic nervous system activity were assessed with an ambulatory electrocardiogram (Lifecard CF, Del Mar Reynolds Medical Inc, Irvine, CA, USA). This system provides ECG data with high accuracy at a sampling frequency of $1,024 \mathrm{~Hz}$ (12-bit resolution). Recordings were performed during experimental conditions and during $24 \mathrm{~h}$. All ECG recordings were beat-by-beat analyzed and interpreted using the Pathfinder Software (Spacelabs Healthcare, Nuremberg, Germany) and manually re-evaluated by an experienced cardiologist (M.W.).

During the 10 min CPTs heart rate was continuously monitored and $15 \mathrm{~s}$ averages were used to provide an accurate heart rate response. $R-R$ intervals were downloaded to Kubios software ${ }^{\circledR}$ and power spectral density was assessed (Niskanen et al. 2004). An advanced smoothness prior approach was applied for detrending $R-R$ intervals using a smoothing parameter of $\lambda=500$, which equals a cut-off frequency of $0.035 \mathrm{~Hz}$ (Tarvainen et al. 2002). During the CPT, 3 min segments of $R-R$ data were recorded prior to and post CWI, and a 1 min segment during CWI (Fig. 1.)
High frequency (HF) power as a marker of vagal activity was analyzed (Task Force 1996; Berntson et al. 1997) and presented in absolute units $\left(\mathrm{ms}^{2}\right)$.In addition, to account for the problem of non-stationarity of $R-R$ data, we used a non-linear method of HRV analysis (detrended fluctuation analysis, DFA) and analyzed the fractal scaling exponent $\left(\alpha_{1}\right) . \alpha_{1}$ describes the fractal correlation properties of $R-R$ interval data (Peng et al. 1995; Tulppo et al. 2005). It is of note that $\alpha_{1}$ has a strong inverse correlation to HF power (Hautala et al. 2003) and we have previously shown that $\alpha_{1}$ follows the same inverse pattern in children during HRV nighttime recordings (Radtke et al. 2013a).

With respect to $24 \mathrm{~h}$ ambulatory electrocardiography, time domain parameters of HRV were analyzed according to current recommendations (Task Force 1996). The device and the electrodes were attached to the chest on a normal school day between 10 a.m. to 12 a.m. and recording was terminated after $24 \mathrm{~h}$. The standard deviation of normal-tonormal intervals (SDNN) and the square root of the mean squared differences of successive normal-to-normal intervals (rMSSD) over $24 \mathrm{~h}$ were calculated (Task Force 1996).

\section{Statistical analysis}

Statistical analyses were performed with SPSS for Windows (Version 17.0, SPSS Inc., Chicago, USA). ShapiroWilk tests were used to check data for normal distribution. $\chi^{2}$, Mann-Whitney $U$ or $t$ tests were performed to compare baseline variables between groups. Normally distributed data are presented as mean \pm standard deviation (SD), and variables with skewed distribution were shown as median (inter-quartile range, IQR). Relations between microvascular endothelial function variables were determined using Spearman's rank correlation. ANCOVAs adjusting for variables differing at baseline were also performed. If the sphericity assumption was violated $(P<0.05)$, the Huynh-Feldt correction was used. A Tukey HSD test was performed to determine significant differences at particular time points in heart rate and BP during the CPT and the hyperemic response during PAT testing between normal reactors and hyperreactors. With respect to HRV analysis, the spectral powers were log transformed (ln). The level of significance was set at $\alpha=0.05$.

\section{Results}

Identification of hyperreactors

Of 378 adolescents in the age group 14-16 years enrolled at the Olten secondary school, of selected classes 68 students volunteered. Fourteen declined study participation and two had to be excluded due to smoking. Finally, 


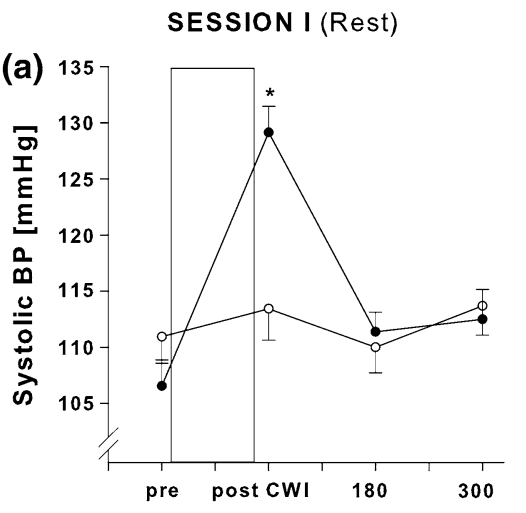

Time Points (s, starting from CWI)

SESSION II (Post Exercise)

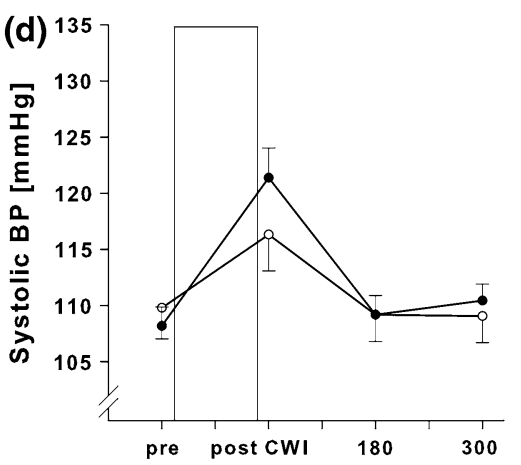

Time Points (s, starting from CWI)

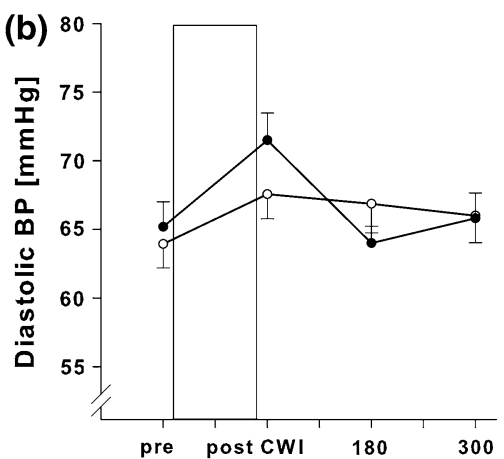

Time Points (s, starting from CWI)

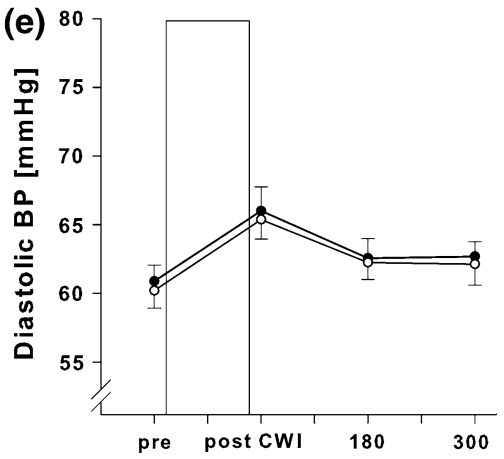

Time Points (s, starting from CWI)

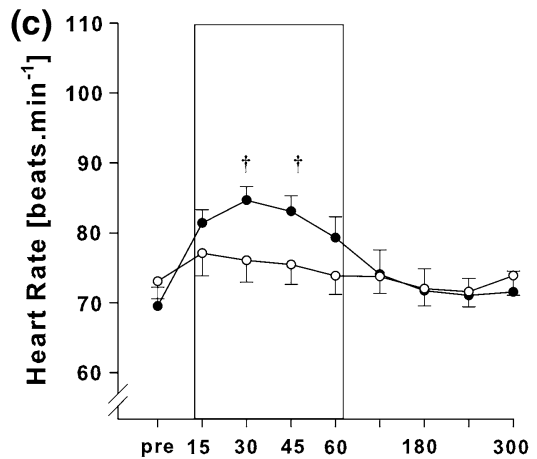

Time Points (s, starting from CWI)

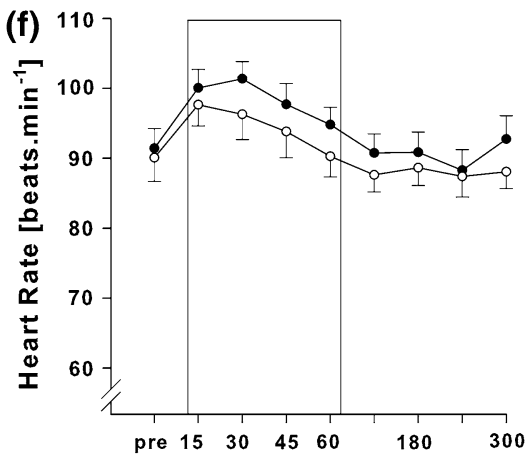

Time Points (s, starting from CWI)
Fig. 2 Cardiovascular response to the cold pressor test at rest (upper graphs) and $30 \mathrm{~min}$ after exhaustive cycling exercise (lower graphs) in hyperreactors and normal reactors. Open circles represent normal reactors and solid circles hyperreactors. The frame indicates the time of cold water immersion (1 min). Please note that the measuring time points between blood pressure (BP) and heart rate differ. $C W I$ coldwater immersion. Data are mean \pm SEM. $* P<0.001$, and ${ }^{\dagger} P<0.05$ indicate differences between normal reactors and hyperreactors at this particular measurement time point

value by $15.2 \pm 8.5$ beats $\min ^{-1}$ to the peak response at 30 s during CWI $(P<0.001)$, while in normal reactors heart rate increased non-significantly by $3.0 \pm 11.8$ beats $\mathrm{min}^{-1}$ $(P=0.326$, Fig. $2 c)$. A group $\times$ time interaction was observed for systolic and diastolic BP, and for heart rate (all $P<0.05$, Fig. 2a-c), indicating a different time course between the two groups. No changes were found in indices of autonomic tone (Fig. 3a, b). During the CPT after exhaustive exercise (session II) significant changes over time were observed for systolic and diastolic BP and heart rate (all $P<0.01$ ) but no group $\times$ time interaction was found (Fig. 2d-f). No between-group differences were observed in markers of HRV (Fig. 3c, d), except higher values for $\alpha_{1}$ in normal reactors prior to CWI $(P=0.042)$.

Impact of BP hyperreactivity on microvascular endothelial function

At rest, median (IQR) baseline PWA in the finger in which the occlusion was performed was significantly
During CWI at rest (session I) significant changes over time were observed for heart rate (all $P<0.05$ ). In hyperreactors, the heart rate increased from the control resting 
Table 1 Description of the study population according to groups of blood pressure reactivity

\begin{tabular}{|c|c|c|c|c|}
\hline & All $(N=32)$ & Hyperreactors $(N=16)$ & Normal reactors $(N=16)$ & $P$ value \\
\hline Age (years) & $14.0(1.0)$ & $14.0(1.0)$ & $14.0(1.0)$ & 0.402 \\
\hline Female sex, $n(\%)$ & $20(63 \%)$ & $10(63 \%)$ & $10(63 \%)$ & 0.642 \\
\hline Tanner stage & & & & 0.673 \\
\hline $4 / 5$ & $32(100 \%)$ & $16(100 \%)$ & $16(100 \%)$ & \\
\hline Stature $(\mathrm{m})$, mean $\pm \mathrm{SD}$ & $1.68 \pm 0.05$ & $1.67 \pm 0.5$ & $1.69 \pm 0.5$ & 0.684 \\
\hline Weight $(\mathrm{kg})$, mean $\pm \mathrm{SD}$ & $57.7 \pm 6.2$ & $57.4 \pm 7.0$ & $58.0 \pm 5.6$ & 0.557 \\
\hline Body mass index $\left(\mathrm{kg} \mathrm{m}^{-2}\right)$, mean $\pm \mathrm{SD}$ & $20.5 \pm 2.1$ & $20.7 \pm 2.4$ & $20.5 \pm 1.9$ & 0.761 \\
\hline Sum of four skinfolds $(\mathrm{mm})$, mean $\pm \mathrm{SD}$ & $46.0 \pm 16.6$ & $47.9 \pm 18.7$ & $44.3 \pm 14.9$ & 0.907 \\
\hline Resting heart rate (beats $\min ^{-1}$ ), mean $\pm \mathrm{SD}$ & $69.4 \pm 10.6$ & $69.7 \pm 10.1$ & $69.7 \pm 10.1$ & 0.418 \\
\hline Systolic BP (z-score), mean \pm SD & $-0.43 \pm 0.9$ & $-0.63 \pm 0.81$ & $-0.25 \pm 0.9$ & 0.211 \\
\hline Diastolic BP ( $z$-score $),$ mean \pm SD & $-0.02 \pm 0.7$ & $0.20 \pm 0.6$ & $-0.53 \pm 0.7$ & 0.854 \\
\hline$\dot{V} \mathrm{O}_{2 \text { peak }}\left(\mathrm{mL} \mathrm{kg}^{-1} \mathrm{~min}^{-1}\right)$, mean $\pm \mathrm{SD}$ & $41.5 \pm 10.0$ & $45.9 \pm 11.2$ & $43.8 \pm 8.5$ & 0.370 \\
\hline Power output (watt $\mathrm{kg}^{-1}$ ), mean $\pm \mathrm{SD}$ & $3.5 \pm 0.5$ & $3.3 \pm 0.6$ & $3.6 \pm 0.5$ & 0.643 \\
\hline Peak heart rate (beats $\min ^{-1}$ ), mean $\pm S D$ & $189.0 \pm 6.7$ & $188.2 \pm 5.1$ & $189.8 \pm 8.0$ & 0.613 \\
\hline $\mathrm{SDNN}(\mathrm{ms})$, mean $\pm \mathrm{SD}$ & $186.1 \pm 40.6$ & $181.7 \pm 47.3$ & $190.6 \pm 33.6$ & 0.546 \\
\hline $\mathrm{rMSSD}(\mathrm{ms})$, mean $\pm \mathrm{SD}$ & $44.2 \pm 12.8$ & $45.4 \pm 13.9$ & $42.9 \pm 11.8$ & 0.597 \\
\hline MVPA (min day ${ }^{-1}$ ), median (IQR) & $36.3(16.7)$ & $36.0(19.2)$ & $36.8(15.8)$ & 0.928 \\
\hline
\end{tabular}

Data are presented as mean $\pm \mathrm{SD}$ or median (IQR) or $N(\%)$

$B P$ blood pressure, MVPA moderate-to-vigorous physical activity, $r M S S D$ root of the mean squared differences of normal-to-normal intervals, $S D N N$ standard deviation of normal-to-normal intervals

A $P$ value $<0.05$ indicates statistical significance

Fig. 3 Comparison of log transformed (ln) high frequency (HF) power (in $\mathrm{ms}^{2}$ ) and the fractal scaling exponent $\alpha_{1}$ during the cold pressor test at rest (upper graphs) and $30 \mathrm{~min}$ after exhaustive cycling exercise (lower graphs). Open circles represent normal reactors and solid circles hyperreactors. The frame indicates the time of cold water immersion (1 min). CWI cold-water immersion. Values are mean \pm SEM. $* P<0.05$
SESSION I (Rest)

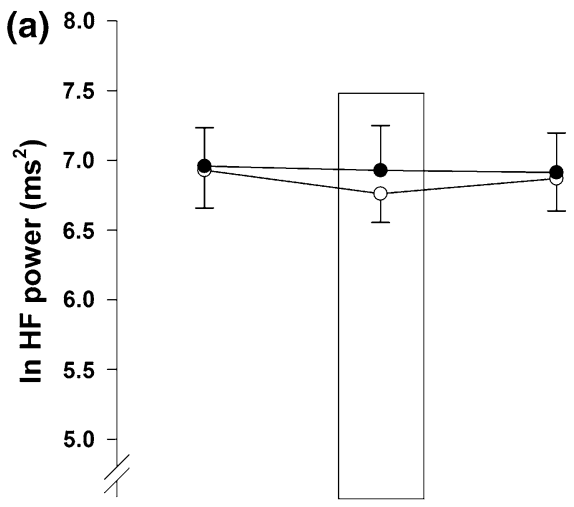

SESSION II (Post exercise)

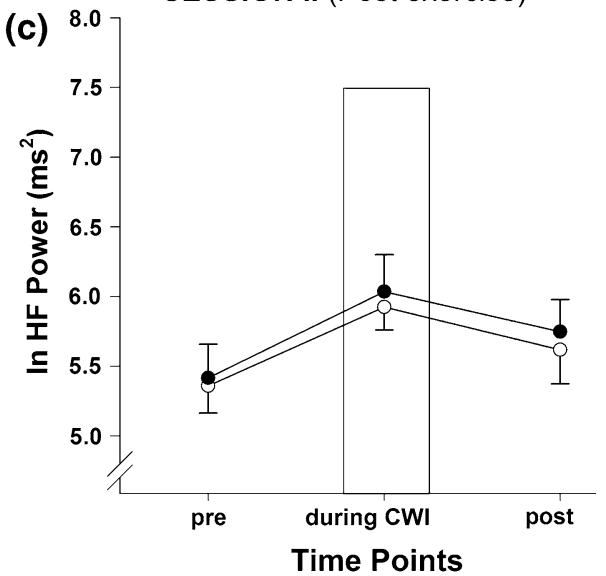

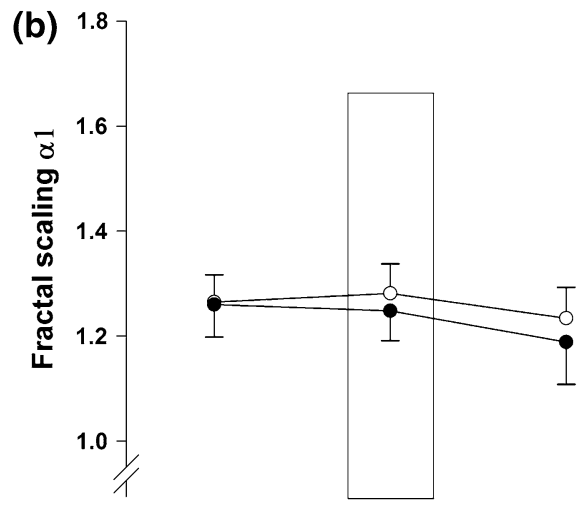

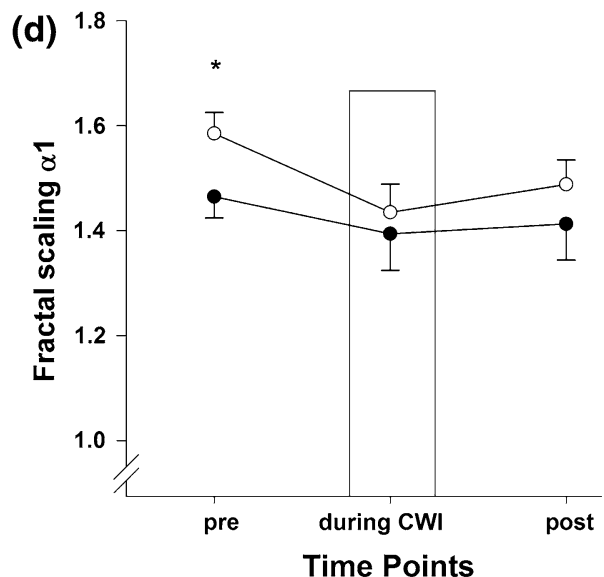


Table 2 Comparison of microvascular endothelial function data measured by peripheral arterial tonometry between hyperreactors and normal reactors

\begin{tabular}{llll}
\hline Variable & Hyperreactor $(N=16)$ & Normal reactor $(N=16)$ & $P$ value \\
\hline RHI rest, mean \pm SD & $2.1 \pm 0.4$ & $1.6 \pm 0.4$ & 0.003 \\
Peak response rest, mean \pm SD & $2.3 \pm 0.6$ & $1.6 \pm 0.5$ & 0.001 \\
RHI post-exercise, median (IQR) & $1.39(0.16)$ & $1.36(0.13)$ & 0.119 \\
Peak response post-exercise, median (IQR) & $1.20(0.29)$ & $1.12(0.28)$ & $202.5 \pm 90.7$ \\
Time to peak (s), mean \pm SD & $100.3 \pm 44.3$ & $225.0(155.0)$ & $<0.491$ \\
Time to peak post-exercise (s), median (IQR) & $130.0(150.0)$ & 0.119 \\
\hline
\end{tabular}

Data are presented as mean \pm SD or median (IQR). Data were analyzed using Student's $t$ test or Mann-Whitney $U$ test, as indicated. The RHI was generated by the software program and the peak response and time to peak were individually determined

$R H I$ reactive hyperemic index

A $P$ value $<0.05$ indicates statistical significance

Fig. 4 Comparison of microvascular endothelial function assessed by peripheral arterial tonometry (PAT) between hyperreactors and normal reactors at rest and after exhaustive cycling exercise. Open circles represent normal reactors and solid circles hyperreactors. Values are mean \pm SEM. $* P<0.05$
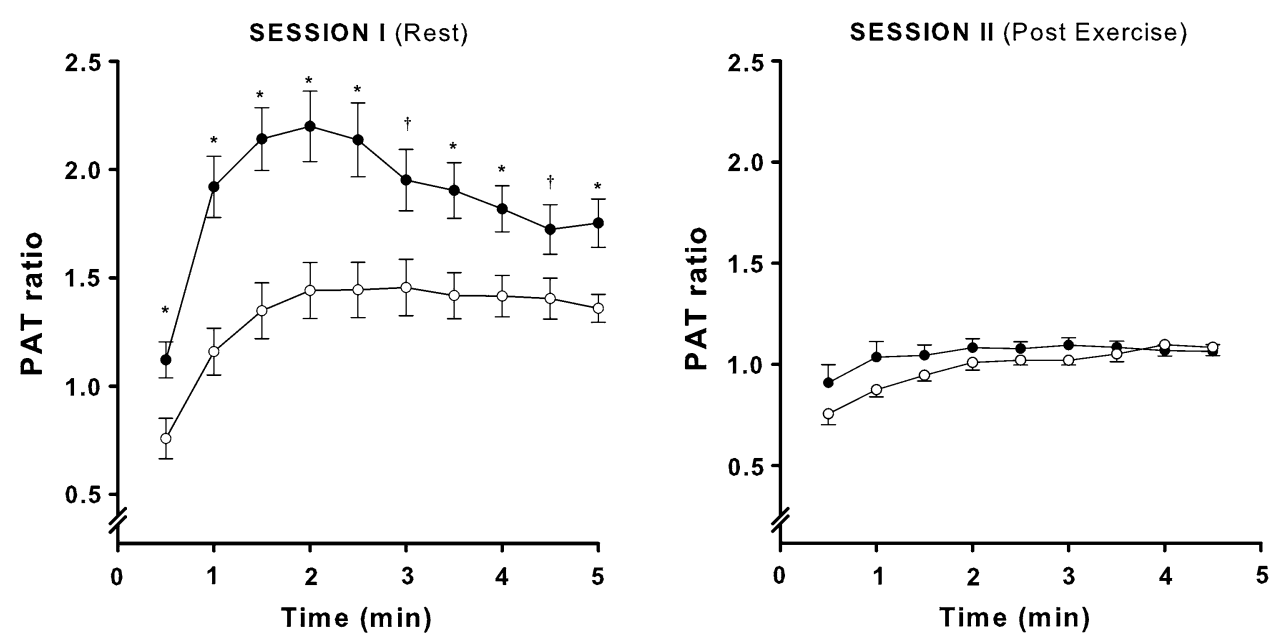

lower in hyperreactors compared with normal reactors [(475.5 (527.9) vs. 192.7 (363.5), $P=0.026]$ with a nonsignificant trend towards lower PWA in the control finger [(503.3 (530.6) vs. 281.05 (369.5), $P=0.077)]$. Baseline PWA were inversely correlated with peak response $(R=-0.696, P<0.001)$. No relationship existed between baseline PWA and neither heart rate nor indices of autonomic tone. Hyperreactors had a significantly higher RHI, peak response and a shorter time to peak in response to ischemia in comparison to normal reactors (see Table 2). Within the ANCOVA model including baseline PWA as a covariate, being a hyperreactor still had a significant impact on PAT ratios $(P=0.041)$. As shown in Fig. 4, the overall hyperemic response curves indicate a higher PAT ratio at all time points in hyperreactors compared to their counterparts. After the acute exercise bout (session II), PWA were significantly higher compared to rest in both groups $(P<0.001)$. No difference was observed between hyperreactors and normal reactors in the occluded finger (747.4 \pm 213.7 vs. $786.7 \pm 215.3, P=0.608)$ and the control finger
$(809.2 \pm 245.5$ vs. $823.9 \pm 203.9, P=0.856)$. RHI and peak response were significantly lower in both groups compared to resting values (all $P<0.05$, Table 2). After exercise, there was no difference between hyperreactors and normal reactors with regard to RHI, peak response, and the PAT ratio (Fig. 4). Figure 5 displays representative PAT recordings performed at rest and post-exercise in a hyperreactive and a normal reactive girl, matched for known predictors of microvascular endothelial function (Radtke et al. 2012).

\section{Discussion}

Results of the present study provide novel insight into BP hyperreactivity and microvascular endothelial function in healthy adolescents. Hyperreactors showed a greater reactivity of the cardiovascular system with a higher RHI, peak response, PAT ratio and time to peak response at rest. Importantly, these differences were abolished after a short bout of maximal exercise. 
Fig. 5 Comparison of peripheral arterial tonometry (PAT) recordings between a hyperreactive and normal reactive girl (both 14 years of age) at rest and after cardiopulmonary exercise testing. Subjects were matched for age, sex, height and Tanner stage. Raw pulse wave amplitudes are displayed at a gain of 200 (Endo-PAT2000 ${ }^{\circledR}$, Itamar Medical Ltd., Israel)

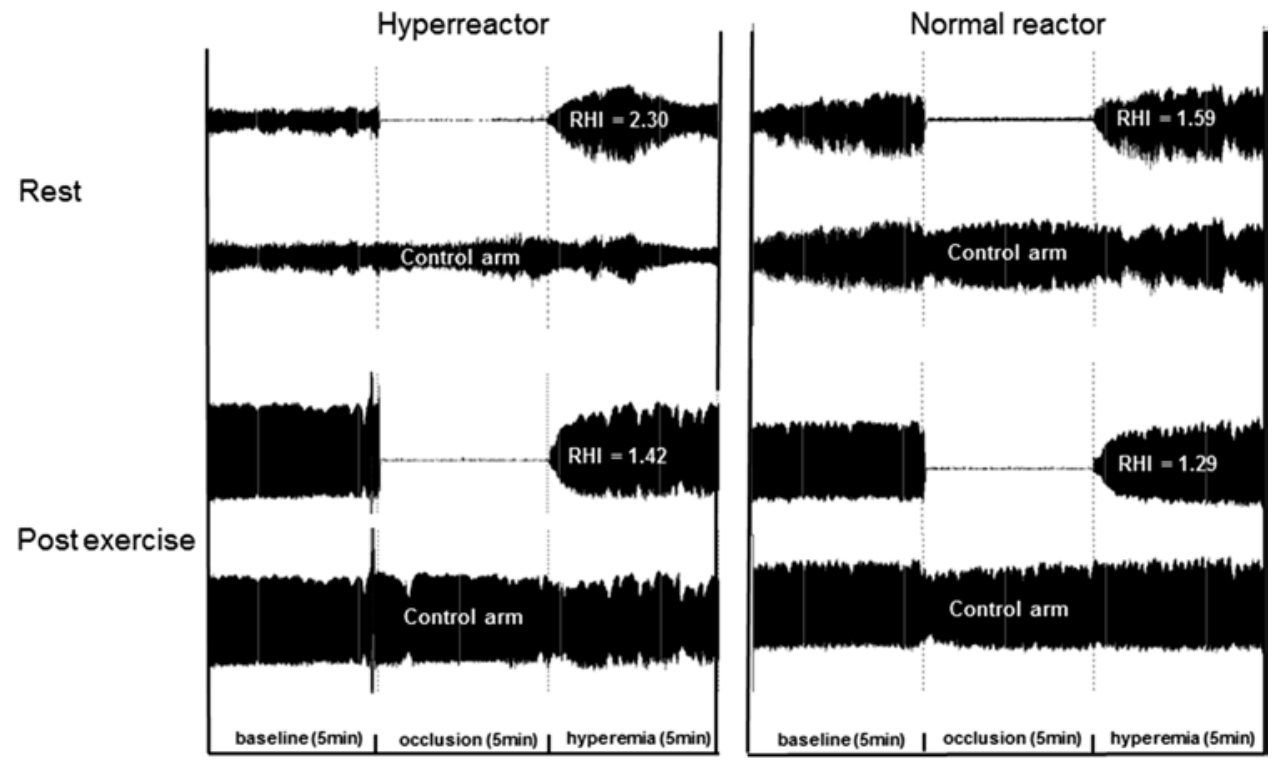

In our random sample of healthy adolescents, $31 \%$ of adolescents were identified as hyperreactors, consistent with previous reports documenting 18-34 \% (Hines 1937; Wood et al. 1984). Hyperreactors showed no differences in anthropometric characteristics, physical activity levels, exercise capacity and indices of autonomic tone compared with normal reactors, supporting the assumption of a strong genetic predisposition for this behavior (Hines 1937; Wood et al. 1984).

In the present study, hyperreactors had a markedly higher RHI at the fingertip compared to normal reactors. It has been shown that adolescent and normotensive hyperreactors are at increased risk of developing hypertension (Wood et al. 1984). In a large cohort of healthy adults, Hamburg et al. (2011) recently observed a positive association between higher BP and RHI using PAT. This group speculated that a higher BP is likely to result in higher hyperemic PWA in the digit and lead to increased microvascular hyperemic reaction. Our data clearly support this assumption in that hyperreactors produce higher PWA in the digits after the application of an ischemic stimulus. These findings appear to be partly driven by lower baseline PWA in hyperreactors and may reflect higher adrenergic activation and as a consequence thereof peripheral vasoconstriction in the fingertips. During data analysis we observed a high inverse Spearman rank correlation coefficient between baseline PWA and RHI, as recently described by others (Lee et al. 2012). We consequently performed an ANCOVA with baseline PWA as a covariate and normal reactors and hyperreactors still significantly differed with regard to PAT ratios, though less significantly $(P=0.041)$.

Peripheral arterial tonometry is an accepted measure of endothelial function and NO dependent (Nohria et al. 2006). The infusion of $N^{\mathrm{G}}$-nitro-L arginine methyl ester
(L-NAME), a NO inhibitor, blunted the PWA response by $46 \%$ (Nohria et al. 2006), indicating that other vasoactive substances contribute to hyperemic PWA changes in the fingertip. Similar to these findings, Coffman (1994) demonstrated that the regulation of finger blood flow is mainly controlled by the autonomic nervous system and to a lesser extent by NO. It is therefore unlikely that the increased hyperemic response during PAT testing at rest is the result of a higher NO bioavailability in the microvasculature of hyperreactors.

The increased vasoreactivity in hyperreactors was merely abolished after a short bout of maximal exercise (session II). Higher PWA were observed in both groups, indicative of post-exercise vasodilation and increased fingertip blood flow. Exercise-induced shear stress and concomitant NO release is likely to contribute to this vasodilation. A possible contribution of the provided snack on post-exercise PWA is unlikely but cannot be excluded. Since there were no between-group differences in baseline PWA post-exercise, we postulate that the increased NO release may have compensated the effect of adrenergic mediated vasoconstriction in hyperreactors observed at rest.

Hyperreactors showed a more pronounced heart rate response during CWI at rest (session I). The increase in heart rate was not accompanied with substantial changes in HF power $\left(\mathrm{ms}^{2}\right)$ as a marker of vagal activity. This suggests that the increase in heart rate was due to adrenergic activation rather than vagal withdrawal, consistent with previous reports in healthy subjects, showing that adrenergic blockade abolishes the increase in heart rate (Victor et al. 1987). After exhaustive cycling exercise, the elevated cardiovascular response to the CPT in hyperreactors was no longer evident. Post-exercise CWI significantly augmented vagal activity. This observation can partially be explained 
by exercise-induced changes in overall sympathetic nervous system activity.

Altogether, cause-effect relationships between hyperreactivity and microvascular endothelial function cannot be explained with the present study. Importantly, $\alpha$ adrenoreceptors in resistance vessels play an important role in the regulation of vascular tone (Van Zwieten et al. 1984), but we can only speculate that receptor responsiveness and/or receptor sensitivity may be altered in hyperreactive subjects. The contribution of NO and the autonomic nervous system in the fingertip hyperemic response of hyperreactors could be a focus of future studies.

Several limitations of this study need to be discussed. Under optimal experimental conditions, spectral power analysis of HRV requires stationary data for periods of 4-5 min. In our setting, shorter time periods were analyzed (1 min segments during CWI) and non-stationarity was likely due to changes in heart rate. Consequently, these data should be interpreted with caution. Nevertheless, spectral power analysis of HF power, as a marker of vagal activity, can be performed with 1 min segments (Berntson et al. 1997; Task Force 1996). Moreover, we used a non-linear method of HRV (DFA), that is not affected by non-stationarity and which revealed comparable results to HF power during the CPT.

Hormonal changes during the female menstrual cycle are known to affect the elastic properties of central arteries (Hayashi et al. 2006). Our experiments were not conducted at a particular time point during the female menstrual cycle, and potential effects of menstrual cycle phase on elastic properties of peripheral arteries in the fingertips using PAT cannot completely be excluded.

\section{Conclusion}

The present study demonstrates that normotensive BP hyperreactors have a higher RHI at the fingertip. PAT is an emerging, easily applicable and operator-independent technique used to assess microvascular endothelial function. However, the operator should be aware of methodological caveats, such as the impact of vascular hyperreactivity on the RHI. Otherwise, vascular hyperreactivity may mimic good vascular endothelial health and therefore bias the interpretation of individual cardiovascular risk using RHI. Especially in studies with small sample sizes and those assessing individual cardiovascular risk, identification of hyperreactors prior to the application of PAT should be considered. Future studies are warranted to elucidate the physiological mechanism linking BP hyperreactivity and microvascular endothelial function preferably in longitudinal designs and in response to repetitive exercise.
Acknowledgments We gratefully acknowledge the subjects' participation in this study and the staff of the Kantonsschule Olten for providing the testing facilities. The study was financially supported by the Olten Heart Foundation.

Conflict of interest The authors declare that they have no competing interests.

\section{References}

Abboud FM, Harwani SC, Chapleau MW (2012) Autonomic neural regulation of the immune system: implications for hypertension and cardiovascular disease. Hypertension 59:755-762

Armstrong N, Williams J, Balding J, Gentle P, Kirby B (1991) The peak oxygen uptake of British children with reference to age, sex and sexual maturity. Eur J Appl Physiol Occup Physiol 62:369-375

Barnett PH, Hines EA Jr, Schirger A, Gage RP (1963) Blood pressure and vascular reactivity to the cold pressor test. Restudy of 207 subjects 27 years later. JAMA 183:845-848

Berntson GG, Bigger JT Jr, Eckberg DL, Grossman P, Kaufmann PG, Malik M, Nagaraja HN, Porges SW, Saul JP, Stone PH, Van der Molen MW (1997) Heart rate variability: origins, methods, and interpretive caveats. Psychophysiology 34:623-648

Bruyndonckx L, Radtke T, Eser P, Vrints CJ, Ramet J, Wilhelm M, Conraads VM (2013) Methodological considerations and practical recommendations for the application of peripheral arterial tonometry in children and adolescents. Int J Cardiol. http://dx. doi.org/10.1016/j.ijcard.2013.07.236

Chen Y, Dangardt F, Osika W, Berggren K, Gronowitz E, Friberg P (2012) Age- and sex-related differences in vascular function and vascular response to mental stress longitudinal and crosssectional studies in a cohort of healthy children and adolescents. Atherosclerosis 220:269-274

Coffman JD (1994) Effects of endothelium-derived nitric oxide on skin and digital blood flow in humans. Am J Physiol Heart Circ Physiol 267:H2087-H2090

Fernandez JR, Redden DT, Pietrobelli A, Allison DB (2004) Waist circumference percentiles in nationally representative samples of African-American, European-American, and Mexican-American children and adolescents. J Pediatr 145:439-444

Flaa A, Eide IK, Kjeldsen SE, Rostrup M (2008) Sympathoadrenal stress reactivity is a predictor of future blood pressure: an 18-year follow-up study. Hypertension 52:336-341

Forte P, Copland M, Smith LM, Milne E, Sutherland J, Benjamin N (1997) Basal nitric oxide synthesis in essential hypertension. Lancet 349:837-842

Gamboa A, Okamoto LE, Diedrich A, Choi L, Robertson D, Farley G, Paranjape S, Biaggioni I (2012) Sympathetic activation and nitric oxide function in early hypertension. Am J Physiol Heart Circ Physiol 302:H1438-H1443. doi:10.1152/ajpheart.01020.2011

Haller MJ, Stein J, Shuster J, Theriaque D, Silverstein J, Schatz DA, Earing MG, Lerman A, Mahmud FH (2007) Peripheral artery tonometry demonstrates altered endothelial function in children with type 1 diabetes. Pediatr Diabetes 8:193-198

Hamburg NM, Palmisano J, Larson MG, Sullivan LM, Lehman BT, Vasan RS, Levy D, Mitchell GF, Vita JA, Benjamin EJ (2011) Relation of brachial and digital measures of vascular function in the community: the Framingham heart study. Hypertension 57:390-396

Hautala AJ, Makikallio TH, Seppanen T, Huikuri HV, Tulppo MP (2003) Short-term correlation properties of $R-R$ interval dynamics at different exercise intensity levels. Clin Physiol Funct Imaging 23:215-223 
Hayashi K, Miyachi M, Seno N, Takahashi K, Yamazaki K, Sugawara J, Yokoi T, Onodera S, Mesaki N (2006) Variations in carotid arterial compliance during the menstrual cycle in young women. Exp Physiol 91:465-472

Hines EA (1937) Reaction of the blood pressure of 400 school children to a standard stimulus. JAMA 108:1249-1250

Hines EA, Brown GE (1936) The cold pressor test for measuring the reactivity of the blood pressure: data concerning 571 normal and hypertensive subjects. Am Heart J 11:1-9

Kasagi F, Akahoshi M, Shimaoka K (1995) Relation between cold pressor test and development of hypertension based on 28-year follow-up. Hypertension 25:71-76

Lee CR, Bass A, Ellis K, Tran B, Steele S, Caughey M, Stouffer GA, Hinderliter AL (2012) Relation between digital peripheral arterial tonometry and brachial artery ultrasound measures of vascular function in patients with coronary artery disease and in healthy volunteers. Am J Cardiol 109:651-657

Mahmud FH, Hill DJ, Cuerden MS, Clarson CL (2009) Impaired vascular function in obese adolescents with insulin resistance. $\mathrm{J}$ Pediatr 155:678-682

Masse LC, Fuemmeler BF, Anderson CB, Matthews CE, Trost SG, Catellier DJ, Treuth M (2005) Accelerometer data reduction: a comparison of four reduction algorithms on select outcome variables. Med Sci Sports Exerc 37:S544-S554

Menkes MS, Matthews KA, Krantz DS, Lundberg U, Mead LA, Qaqish B, Liang KY, Thomas CB, Pearson TA (1989) Cardiovascular reactivity to the cold pressor test as a predictor of hypertension. Hypertension 14:524-530

Morris NM, Udry JR (1980) Validation of a self-administered instrument to assess stage of adolescent development. J Youth Adolesc 9:271-280

National High Blood Pressure Education Program Working Group on High Blood Pressure in Children and Adolescents (2004) The fourth report on the diagnosis, evaluation, and treatment of high blood pressure in children and adolescents. Pediatrics 114:555-576

Niskanen JP, Tarvainen MP, Ranta-Aho PO, Karjalainen PA (2004) Software for advanced HRV analysis. Comput Methods Programs Biomed 76:73-81

Nohria A, Gerhard-Herman M, Creager MA, Hurley S, Mitra D, Ganz $\mathrm{P}$ (2006) Role of nitric oxide in the regulation of digital pulse volume amplitude in humans. J Appl Physiol 101:545-548

Paridon SM, Alpert BS, Boas SR, Cabrera ME, Caldarera LL, Daniels SR, Kimball TR, Knilans TK, Nixon PA, Rhodes J, Yetman
AT (2006) Clinical stress testing in the pediatric age group: a statement from the American Heart Association Council on Cardiovascular Disease in the Young, Committee on Atherosclerosis, Hypertension, and Obesity in Youth. Circulation 113:1905-1920

Peng CK, Havlin S, Stanley HE, Goldberger AL (1995) Quantification of scaling exponents and crossover phenomena in nonstationary heartbeat time series. Chaos 5:82-87

Radtke T, Khattab K, Eser P, Kriemler S, Saner H, Wilhelm M (2012) Puberty and microvascular function in healthy children and adolescents. J Pediatr 161:887-891

Radtke T, Khattab K, Brugger N, Eser P, Saner H, Wilhelm M (2013a) High-volume sports club participation and autonomic nervous system activity in children. Eur J Clin Invest. doi:10.1111/eci.12112

Radtke T, Kriemler S, Eser P, Saner H, Wilhelm M (2013b) Physical activity intensity and surrogate markers for cardiovascular health in adolescents. Eur J Appl Physiol 113:1213-1222

Selamet Tierney ES, Newburger JW, Gauvreau K, Geva J, Coogan E, Colan SD, de Ferranti SD (2009) Endothelial pulse amplitude testing: feasibility and reproducibility in adolescents. J Pediatr 154:901-905

Tarvainen MP, Ranta-Aho PO, Karjalainen PA (2002) An advanced detrending method with application to HRV analysis. IEEE Trans Biomed Eng 49:172-175

Task Force of the European Society of Cardiology and the North American Society of Pacing and Electrophysiology (1996) Heart rate variability: standards of measurement, physiological interpretation and clinical use. Circulation 93:1043-1065

Treuth MS, Schmitz K, Catellier DJ, McMurray RG, Murray DM, Almeida MJ, Going S, Norman JE, Pate R (2004) Defining accelerometer thresholds for activity intensities in adolescent girls. Med Sci Sports Exerc 36:1259-1266

Tulppo MP, Kiviniemi AM, Hautala AJ, Kallio M, Seppanen T, Makikallio TH, Huikuri Heikki V (2005) Physiological background of the loss of fractal heart rate dynamics. Circulation 112:314-319

Van Zwieten PA, Timmermans PB, Brummelen P (1984) Role of alpha adrenoreceptors in hypertension and in antihypertensive drug treatment. Am J Med 77:17-25

Victor RG, Leimbach WN Jr, Seals DR, Wallin BG, Mark AL (1987) Effects of the cold pressor test on muscle sympathetic nerve activity in humans. Hypertension 9:429-436

Wood DL, Sheps SG, Elveback LR, Schirger A (1984) Cold pressor test as a predictor of hypertension. Hypertension 6:301-306 